\title{
A fuzzy index model for trophic status evaluation of reservoir waters
}

\author{
Yi-Ting Liou,*, Shang-Lien $\mathrm{Lo}^{\mathrm{b}}$ \\ ${ }^{a}$ Department of Business Administration, Kainan University, No. 1 Kainan Road, Luzhu, Taoyuan County 338, Chinese Taiwan \\ ${ }^{\mathrm{b}}$ Graduate Institute of Environmental Engineering, National Taiwan University, No. 71 Zoushan Road, Taipei 106, Chinese Taiwan
}

Available online 23 March 2005

\begin{abstract}
An index model for quality evaluation based on the formula of similarity membership functions in the fuzzy c-means (FCM) clustering algorithm is proposed. Summing up the weighted similarity degrees between an observation and designed specific quality-ordered levels develops an alternative overall index. Stretching the values of the controlling parameters in the formula of the similarity membership functions causes diverse patterns of overall index models. Applying this proposed fuzzy index model to the trophic evaluation of reservoir waters is studied to demonstrate the practical application of this index. Every measurement of the variables is standardized by the membership function of quality evaluation on the interval $[0,1]$, referring to the trophic status clarified in the Carlson Trophic State Index. The sensitivity analyses are studied both in the proposed index system and the Carlson Trophic State Index. Besides, a case study of the trophic status evaluation of the Feitsui Reservoir from 1987 to 2003 is presented to demonstrate the feasibility of applying the proposed evaluation model.
\end{abstract}

(C) 2005 Elsevier Ltd. All rights reserved.

Keywords: Environmental-quality assessment; Fuzziness index; Fuzzy c-means clustering algorithm; Similarity membership function; Trophic status of reservoirs

\section{Introduction}

Fuzzy set theory, which introduced the specification of uncertainty by membership functions, has been used to evaluate quality. Silvert $(1997,2000)$ stated that fuzzy logic could be applied to the development of environmental indices in a manner that solves several common problems, including the incompatibility of observations and the need for implicit value judgments. Fuzzy synthetic evaluation and fuzzy cluster analysis have been studied and used in environmental-quality evaluation since the 1990s. For example, Lu et al. (1999)

\footnotetext{
*Corresponding author. Tel.: + $88633412500 \times 1021$; fax: +88623928821 .

E-mail address: ytliou@mail.knu.edu.tw (Y.-T. Liou).
}

studied the feasibility of applying fuzzy synthetic evaluation to the quality of water in the Feitsui Reservoir in Taiwan. According to the results, fuzzy synthetic evaluation does a better job of detecting shortterm changes of water quality and exploring the overturning phenomena than the Carlson Index. Chang et al. (2001) assessed water-quality conditions using three fuzzy synthetic evaluation approaches and compared the results with the conventional Water Quality Index for rivers. Fuzzy synthetic evaluation generally uses a numerical scale to represent water quality and provides an alternative methodology for aggregating the values of the parameters to various quality features. Nonetheless, contradictions arise in fuzzy synthetic analysis when any two of the critical parameters incline extremely to different strengths of the evaluated status. Rather than 
assigning a numerical scale to water quality, fuzzy classification systems categorize water bodies into several quality classes or levels. For example, Kung et al. (1992) used fuzzy clustering analysis to evaluate the quality of water in Dianshan Lake near Shanghai, China. Melcher and Matthies (1996) applied fuzzy clustering to data on phytotoxicity. Tao and Xinmiao (1998) estimated the quality of an urban traffic environment in Anshan, China, with fuzzy clustering analysis.

Alternatively, the popularity of fuzzy c-means (FCM) clustering algorithms has grown considerably among researchers in diverse fields because of the algorithms' versatility and the ease with which they can be adapted to the requirements of different research communities. Bezdek (1980) provided an excellent treatise on the family of FCM methods in 1980. Furthermore, the convergence analysis of the FCM algorithm has attracted much attention since the publication of Bezdek's convergent theory in 1978 (Bezdek, 1980; Fadili et al., 2001). Hammah and Curran (1999) stated that the FCM clustering algorithm constitutes a more computationally attractive alternative to the applications in the field of water resources. An index model based on the formula of the similarity-membership function in Bezdek's convergent theory was initiated in Liou et al. (2003) on a river quality evaluation that involved mathematical-modeling and knowledge bases. This paper further studies the application of the fuzzy index system in the field of environmental quality assessment, the manipulation of controlling parameters, the sensitivity analysis of the model, the application on reservoir water quality, and the evaluation of trophic status.

\section{Materials and methods}

\subsection{The convergent formula of fuzzy c-means}

In principle, the convergent formula of FCM could be obtained by using Picard interaction between updated prototypes $\vec{v}_{i}$ and updated similarity degrees $\mu_{k i}$, where $\mu_{k i}$ denotes the similarity degree between an object data $\vec{x}_{k}$ and the prototype $\vec{v}_{i}$ (Fadili, 2001; Hammah and Curran, 1999; Flores-Sintas et al., 1999; Whitman and Fahringer, 1973). Based on the convergent theory of FCM, the similarity measurement of two sets is revealed to the following expressions:

$$
\begin{aligned}
& \mu_{k i}=\frac{1}{\sum_{j=1}^{c}\left[\left\|\vec{x}_{k}-\vec{v}_{i}\right\|_{A}^{2} /\left\|\vec{x}_{k}-\vec{v}_{j}\right\|_{A}^{2}\right]^{1 / m-1}}, \\
& \mu_{k i}=1, \quad 1 \leqslant i \leqslant c \quad \text { if }\left\|\vec{x}_{k}-\vec{v}_{i}\right\|_{A}^{2}=0,
\end{aligned}
$$

$\mu_{k i} \in[0,1]$ and $\sum_{j=1}^{c} \mu_{k j}=1$,

where $c$ represents numbers of prototypes; $m \in[1, \infty]$, which is called the fuzziness index, regulates the degree between similarity measures and distance measures; $\left\|\vec{x}_{k}-\vec{v}_{i}\right\|_{A}^{2}$ denotes distance functions and when the covariance matrix of all the observations in the data set is equivalent to the identity matrix $I$, then the distance metric becomes equivalent to the Euclidean distance norm. Eq. (1a) regards the similarities between each observation and the groups of prototypes and demonstrates the specific rationale of the measurement between the similarity and distance for two points.

\subsection{The index model for quality evaluation}

The index model for quality evaluation is used to denote $f_{k}$ as the set of corresponding measurements of quality membership degree if $\vec{x}_{k}$ represents the value of a set of an observation and takes a value between zero and one. The quality memberships are used for standardizing the various natural measurement scales into a measurement of the quality degrees (Silvert, 1997,2000; Liou et al., 2003). A fuzzy index model for quality evaluation, based on the convergent theory of FCM (as shown in Eq. (1)); is generated. The Euclidean distance is assigned for measuring the distance between pre-processed object data $\vec{f}_{k}$ and the prototype $\vec{e}_{i}$. The prototype $\vec{e}_{i}$ defines the specific quality-ordered levels. The similarity measures quantify the commonality between an observation and the groups of the assigned specific quality-ordered levels, so an overall quality index of an observation $\left(\vec{x}_{k}\right)$ can be obtained by summing all of its similarities (Liou et al., 2003). The Fuzzy Environmental Quality Index $\left(\mathrm{EQI}_{\mathrm{F}}\right)$ is generally named for the index system applying to the environmental quality assessment. The formula for $\mathrm{EQI}_{\mathrm{F}}$ is shown in Eq. (2)

$$
\begin{aligned}
& \mathrm{EQI}_{\mathrm{F}}=\left(\sum_{i=1}^{c} \mu_{k i} \times q_{i}\right) \times 100, \\
& \mu_{k i}=\frac{1 /\left(\left\|\vec{f}_{k}-\vec{e}_{i}\right\|^{2 /(m-1)}\right)}{\sum_{j=1}^{c}\left[1 /\left(\left\|\vec{f}_{k}-\vec{e}_{j}\right\|^{2 /(m-1)}\right)\right]}, \\
& \mu_{k i}=1, \quad 1 \leqslant i \leqslant c \quad \text { if }\left\|\vec{f}_{k}-\vec{e}_{i}\right\|^{2}=0, \\
& \mu_{k i} \in[0,1] \quad \text { and } \quad \sum_{j=1}^{c} \mu_{k j}=1 .
\end{aligned}
$$

The index of an observation is governed by two variables: the value of the fuzziness index and the number of specific quality-ordered levels. The specific quality level scores, $q_{i} \in[0,1]$, are assigned according to 
the number of specific quality-ordered levels. For example, three specific quality-ordered levels would be given as $q_{1}=0, q_{2}=0.5$, and $q_{3}=1$. Four plausible characteristics of the proposed index model can be described based on Eq. (2). First, the similarity degree between a transferred observation $\left(f_{k}\right)$ and the level of completely perfect quality status declines as the amount of the pollutants increases; but the similarity between the transferred observation $\left(f_{k}\right)$ and the level of completely poor quality increases as the quality of the observation decreases. Second, the number of similarity memberships increase with the number of specific qualityordered levels. Third, as one of the values of the distances between a transferred observation and any one of the specific quality-ordered levels, $\left\|f_{k}-\vec{e}_{i}\right\|^{2}$ is zero, then values of the similarity degrees, $\mu_{k i}$, are only zero or one. Fourth, the $\mathrm{EQI}_{\mathrm{F}}$ is converted to the value on an interval scale from 0 to 100 (worst-to-best quality). The registered $\mathrm{EQI}_{\mathrm{F}}$ is consecutively changed with the quality of the observations. A greater commonality between an observation and good quality level corresponds to a higher overall score.

\subsection{The trophic-status evaluation of reservoir waters}

In Taiwan, reservoirs are the principle infrastructures for storing fresh water from the island's steep mountains and rapidly flowing rivers. Taiwan has a total of around 40 reservoirs. Multivariable indexing allows for a more thorough investigation of water quality and a more continuous description of the eutrophication process than a single variable index system ( $\mathrm{Lu}$ and Lo, 2002). The Carlson Trophic State Index (CTSI) (Carlson, 1977) is currently used by the Taiwan's Environmental Protection Administration to explore monitoring trends for both planning and day-to-day management of reservoirs for the public. The CTSI's classification system, however, has been criticized. Values between
41 and 49 are classified as mesotrophic status, while those equal to 50 or higher than 50 are given as eutrophic status. The index regards CTSI $=49$ and 50 as different trophic states, although the differences between the two are small, but CTSI $=41$ and 49 as the same trophic states, even though the differences between them are large (Lu et al., 1999). The proposed index model provides an alternative index system to evaluate the trophic status of the reservoirs. It is noted that instead of $\mathrm{EQI}_{\mathrm{F}}$ denoted above, the Fuzzy Eutrophic Index $\left(\mathrm{EUI}_{\mathrm{F}}\right)$ is named for trophic status evaluation. A case study of the Feitsui Reservoir is presented to illustrate the application and advantages of the EUI $\mathrm{F}_{\mathrm{F}}$.

\section{Results and discussion}

\subsection{Manipulating the fuzziness index and numbers of specific quality-ordered levels}

A group of synthetic data sets are generated for modeling the results of $\mathrm{EQI}_{\mathrm{F}}$ by manipulating the fuzziness index and numbers of specific quality-ordered levels. A total of 101 transferred four-dimension subsets, ranging from the terminal values $f_{0}=(0,0,0,0)$ to $f_{101}=(1,1,1,1)$ with intervals of 0.01 , are used to compute the similarity degree and the final overall index, $\mathrm{EQI}_{\mathrm{F}}$. Parts of similarity degrees and $\mathrm{EQI}_{\mathrm{F}}$ values with the condition of $(m, c)=(2,3)$ are listed in Table 1. Also in Table 1 , the similarity degree $\mu_{k 1}, \mu_{k 2}$ and $\mu_{k 3}$ of the synthetic data set $f_{k}$ are derived separately by

$$
\mu_{k i}=\frac{1 /\left\|\vec{f}_{k}-\vec{e}_{i}\right\|^{2}}{\sum_{j=1}^{3}\left[1 /\left(\left\|\vec{f}_{k}-\vec{e}_{j}\right\|^{2}\right)\right]}
$$

after imputing the specific quality-ordered levels as $\vec{e}_{1}=$ $(0,0,0,0), \vec{e}_{2}=(0.5,0.5,0.5,0.5)$ and $\vec{e}_{3}=(1,1,1,1)$. Thus, a group of three similarity memberships for each

Table 1

The results of similarity memberships $\left(\mu_{\mathrm{k} 1}, \mu_{\mathrm{k} 2}, \mu_{\mathrm{k} 3}\right)$ and $\mathrm{EQI}_{\mathrm{F}}$ values for parts of the synthetic data under the condition of $(m, c)=(2,3)$

\begin{tabular}{lllll}
\hline$\vec{f}_{k}$ & $\mu_{k 1}$ & $\mu_{k 2}$ & $\mu_{k 3}$ & $\mathrm{EQI}_{\mathrm{F}}:\left(0 \times \mu_{k 1}+0.5 \times \mu_{k 2}+1 \times \mu_{k 3}\right) \times 100$ \\
\hline $\mathbf{( 0 . 0 0 , 0 . 0 0 , 0 . 0 0 , 0 . 0 0 )}$ & $\mathbf{1}$ & $\mathbf{0}$ & $\mathbf{0}$ & $\mathbf{0 . 0 0}$ \\
$(0.10,0.10,0.10,0.10)$ & 0.93 & 0.06 & 0.01 & 4.06 \\
$(0.20,0.20,0.20,0.20)$ & 0.66 & 0.29 & 0.04 & 18.89 \\
$(0.30,0.30,0.30,0.30)$ & 0.29 & 0.66 & 0.05 & 38.11 \\
$(0.40,0.40,0.40,0.40)$ & 0.06 & 0.92 & 0.03 & 48.41 \\
$(\mathbf{0 . 5 0 , 0 . 5 0 , 0 . 5 0 , 0 . 5 0 )}$ & $\mathbf{0}$ & $\mathbf{1}$ & $\mathbf{0}$ & $\mathbf{5 0 . 0 0}$ \\
$(0.60,0.60,0.60,0.60)$ & 0.03 & 0.92 & 0.06 & 51.59 \\
$(0.70,0.70,0.70,0.70)$ & 0.05 & 0.66 & 0.29 & 61.89 \\
$(0.80,0.80,0.80,0.80)$ & 0.04 & 0.29 & 0.66 & 81.11 \\
$(0.90,0.90,0.90,0.90)$ & 0.01 & 0.06 & 0.93 & 95.94 \\
$(\mathbf{1 . 0 0}, \mathbf{1 . 0 0}, \mathbf{1 . 0 0}, \mathbf{1 . 0 0})$ & $\mathbf{0}$ & $\mathbf{0}$ & $\mathbf{1}$ & $\mathbf{1 0 0 . 0 0}$ \\
\hline
\end{tabular}


subset can be calculated. The $\mathrm{EQI}_{\mathrm{F}}$ is aggregated according to summing up the similarity degree, $\mathrm{EQI}_{\mathrm{F}}=$ $\left(\mu_{k 1} \times 0+\mu_{k 2} \times 0.5+\mu_{k 3} \times 1\right) \times 100$. Obviously, the $\mathrm{EQI}_{\mathrm{F}}$ is converted to the value from $\_0$ to 100 along with the changes of synthetic data set $f_{k}$ from worst to best quality. In addition, Fig. 1 shows the results of the similarity degrees and $\mathrm{EQI}_{\mathrm{F}}$ values with the condition of $(m, c)=(2,2)$ for all of the 101 synthetic data sets, where the specific quality-ordered levels are assigned as $\vec{e}_{1}=$ $(0,0,0,0)$ and $\vec{e}_{2}=(1,1,1,1)$. It is obvious that as the value of the synthetic data sets $f_{k}$ increased, the similarity degree $\mu_{k 2}$ increases, but the similarity degree $\mu_{k 1}$ decreases. In the other words, the degree of similarity between the observation and the level of poor quality decreases as the quality of the observation increases. In Fig. 1 the curve of EQI $\mathrm{F}_{\mathrm{F}}$ overlaps with $\mu_{k 2}$ because $\mathrm{EQI}_{\mathrm{F}}$ is derived from $\mathrm{EQI}_{\mathrm{F}}=\left(\mu_{k 1} \times 0+\mu_{k 2} \times\right.$ 1) $\times 100$.

Fig. 2 shows the results of $\mathrm{EQI}_{\mathrm{F}}$ by varying the fuzziness index and the number of specific qualityordered levels. A total of 12 index curves are generated under the condition of setting the fuzziness index to 5,3 , 2 , and $3 / 2$, and the specific quality-ordered level to 2,3 , and 5. A lower fuzziness index corresponds to crisper similarity membership grades. The right side of Fig. 2 shows that the distribution of the $\mathrm{EQI}_{\mathrm{F}}$ is closed to the hard partition. Any observation can be easily assigned to one of the specific quality levels. Besides, the left side of Fig. 2 shows that when the synthetic data set $f_{k}$ approximately coincides with any of the specific qualityordered levels, values of $\mathrm{EQI}_{\mathrm{F}}$ might increase with decreasing quality or decline with increasing quality. The discrepancy in value of $\mathrm{EQI}_{\mathrm{F}}$ is explicit while a high

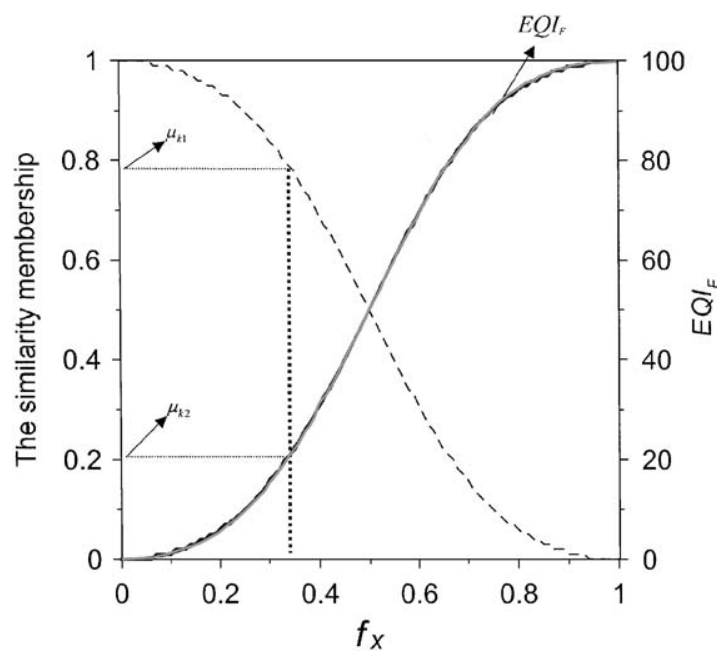

Fig. 1. The results of similarity memberships and $\mathrm{EQI}_{\mathrm{F}}$ values for the 101 synthetic data sets under the condition of $(m$, $c)=(2,2)$. Only two curves are shown because the curve of $\mathrm{EQI}_{\mathrm{F}}$ overlaps with $\mu_{k 2}$. value of fuzziness index associates with excess specific quality-ordered levels, as the index curves shown in Figs. 2 (C, I) and 3 (C, II). An excess specific quality-ordered level causes the unreasonable relationship between observations and the overall index, and leads to contradiction in consecutive analysis. The index model switches from fuzzy theory to crisp theory as the value of data point coincides with the value of the specific quality-ordered levels. Moreover, the $\mathrm{EQI}_{\mathrm{F}}$ yields the average of the distance of a data set to specific qualityordered levels under the condition of $c=2, m=3$, so that the linear relationship between the subset of synthetic data $\vec{f}_{k}$ and $\mathrm{EQI} \mathrm{I}_{\mathrm{F}}$ exists perfectly (Liou et al., 2003). Furthermore, $m=2$ is recommended for consecutive trend analysis for no matter how many specific quality-ordered levels are assigned, the contradiction could be prevented.

\subsection{Applying the index model to trophic-status evaluation of reservoir waters}

The three critical parameters - the concentration of total phosphorous, the concentration of chlorophyll- $a$, and the depth of Secchi disk are employed to evaluate trophic status ( $\mathrm{Lu}$ et al., 1999; $\mathrm{Lu}$ and Lo, 2002; Carlson, 1977; OECD, 1982; Chang and Chuang, 2001). Let $X=\left\{x_{\mathrm{tp}}, x_{\text {chla }}, x_{\mathrm{sd}}\right\}$ be a three-dimensional sampling space for the evaluation of trophic status, and have tp denote the concentration of total phosphorous $(\mu \mathrm{g} / \mathrm{L})$, chla the concentration of chlorophyll- $a(\mu \mathrm{g} / \mathrm{L})$, and sd Secchi disk depth (m). Three crucial breakpoints $1 / 3,1 /$ 2 , and $2 / 3$ are registered in accordance with Carlson Trophic State Index (CTSI) system (shown in Table 2). The segmented linear membership functions of each critical variable are generated as follows:

$$
f_{\mathrm{tp}}\left(x_{\mathrm{tp}}\right)= \begin{cases}1 & \text { for } x_{\mathrm{tp}}>36, \\ (2 / 3)+(1 / 3) \frac{x_{\mathrm{tp}}-24}{36-24} & \text { for } 24<x_{\mathrm{tp}} \leqslant 36, \\ (1 / 3)+(1 / 3) \frac{x_{\mathrm{tp}}-12}{24-12} & \text { for } 12<x_{\mathrm{tp}} \leqslant 24, \\ (1 / 3) \frac{x_{\mathrm{tp}}}{12-0} & \text { for } x_{\mathrm{tp}} \leqslant 12,\end{cases}
$$

$$
\begin{aligned}
& f_{\text {chla }}\left(x_{\text {chla }}\right) \\
& = \begin{cases}1 & \text { for } x_{\text {chla }}>9.8, \\
(2 / 3)+(1 / 3) \frac{x_{\text {chla }}-7.2}{9.8-7.2} & \text { for } 7.2<x_{\text {chla }} \leqslant 9.8, \\
(1 / 3)+(1 / 3) \frac{x_{\text {chla }}-2.6}{7.2-2.6} & \text { for } 2.6<x_{\text {chla }} \leqslant 7.2, \\
(1 / 3) \frac{x_{\text {chla }}}{2.6-0} & \text { for } x_{\text {chla }} \leqslant 2.6,\end{cases} \\
& f_{\text {sd }}\left(x_{\mathrm{sd}}\right)= \begin{cases}(2 / 3)+(1 / 3) \frac{2-x_{\text {sd }}}{2-0} & \text { for } x_{\mathrm{sd}}<2, \\
(1 / 3)+(1 / 3) \frac{4-x_{\mathrm{sd}}}{4-2} & \text { for } 4<x_{\mathrm{sd}} \leqslant 2, \\
(1 / 3) \frac{6-x_{\mathrm{sd}}}{6-4} & \text { for } 6<x_{\mathrm{sd}} \leqslant 4, \\
0 & \text { for } x_{\mathrm{sd}} \geqslant 6 .\end{cases}
\end{aligned}
$$




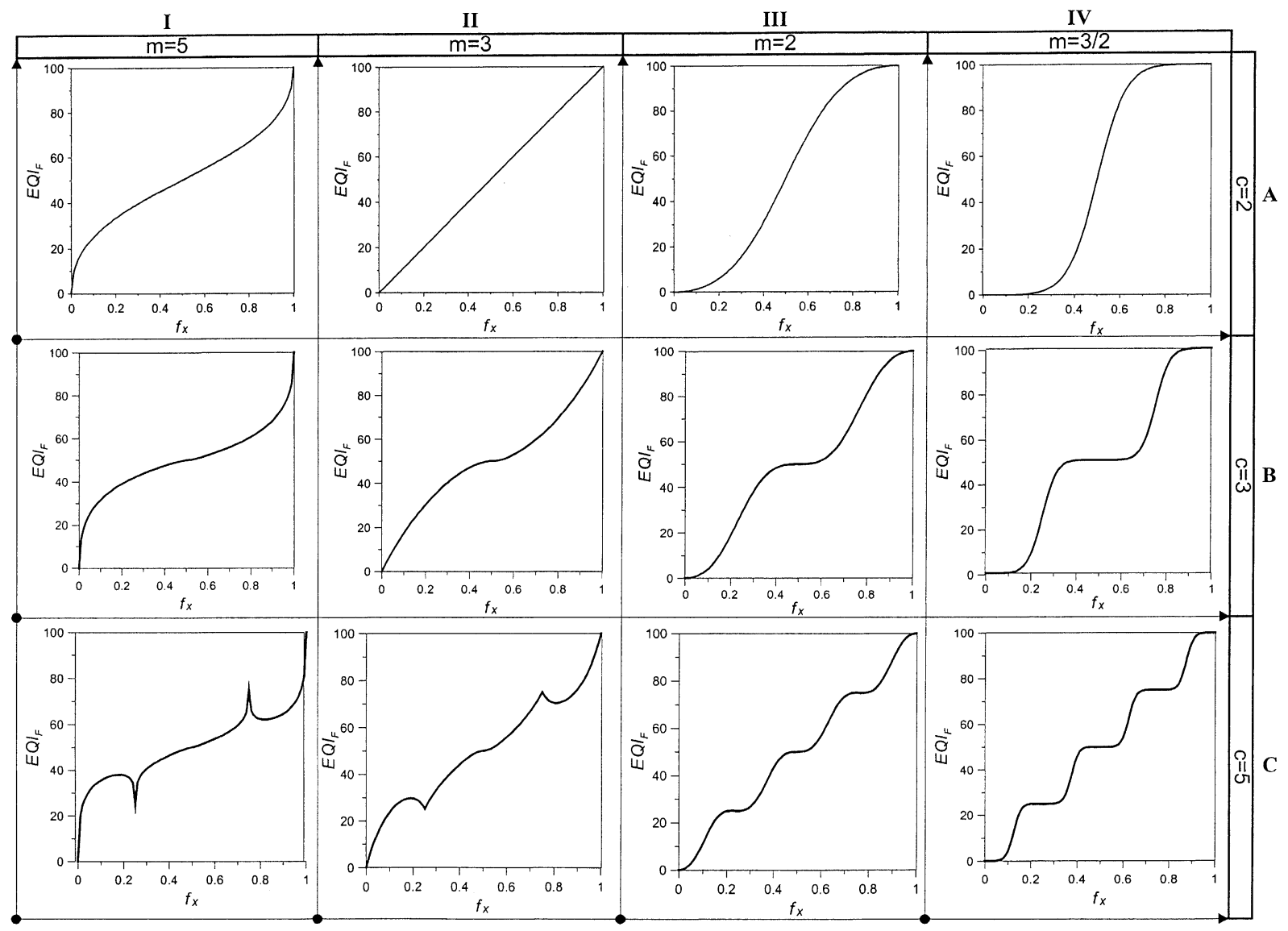

Fig. 2. The EQI $I_{F}$ curves generated under the condition of varying the fuzziness index and the specific quality-ordered levels.

Table 2

The key-points for determining trophic status based on Carlson's Trophic State Index (CTSI) system

\begin{tabular}{lccc}
\hline $\begin{array}{l}\text { Crucial breakpoint } \\
\text { of eutrophicated } \\
\text { membership value }\end{array}$ & $x_{\mathrm{tp}}(\mu \mathrm{g} / \mathrm{L})$ & $x_{\text {chla }}(\mu \mathrm{g} / \mathrm{L})$ & $x_{\mathrm{sd}}(\mathrm{m})$ \\
\hline $1 / 3$ & $<12$ & $<2.6$ & $>4$ \\
$1 / 2$ & 17 & 4.34 & 2.83 \\
$2 / 3$ & $>24$ & $>7.2$ & $<2$ \\
\hline
\end{tabular}

Here, $f_{\text {tp }}, f_{\text {chla }}$, and $f_{\text {sd }}$ represent the transferred measurement. Fig. 3 shows the distribution of the membership function on the interval $[0,1]$.

The two specific eutrophic-ordered levels are defined as $\vec{e}_{1}=\left(f_{\text {tp }}, f_{\text {chla }}, f_{\text {sd }}\right)=(0,0,0)$ and $\vec{e}_{2}=$ $\left(f_{\mathrm{tp}}, f_{\text {chla }}, f_{\mathrm{sd}}\right)=(1,1,1)$ for revealing against time to the consecutive trend analysis. The $\mathrm{EUI}_{\mathrm{F}}$, named for trophic status evaluation, is calculated by $\mathrm{EUI}_{\mathrm{F}}=$ $\left(\mu_{k 1} \times 0+\mu_{k 2} \times 1\right) \times 100$. The term $\mu_{k 1}$ represents the similarity between the observation $\vec{x}_{k}$ and the oligotrophic status; $\mu_{k 2}$ indicates the similarity between the observation and the eutrophic status. The similarity between the observation and the assigned trophic status is derived as

$\mu_{k i}=\frac{1 /\left[\left\|\vec{f}_{k}-\vec{e}_{i}\right\|^{2 /(m-1)}\right]}{\sum_{j=1}^{2}\left[1 /\left(\left\|\vec{f}_{k}-\vec{e}_{j}\right\|^{2 /(m-1)}\right)\right]}$,

where $\vec{f}_{k}$ represents the vector of the transferred observation. The value of $\mathrm{EUI}_{\mathrm{F}}$ is high when the eutrophic status rises. Sensitivity analysis reveals how the value of the index changes along with the observation. Each of the criteria parameters is varied over its possible transition interval respectively, while the others are fixed at the initiation. The sensitivity analysis is inspected by initially setting $\vec{x}_{k}=\left(x_{\mathrm{tp}}, x_{\text {chla }}, x_{\mathrm{sd}}\right)=$ $(17,4.34,2.83)$, where the CTSI $=45$ and the transferred vector $f_{k}=(1 / 2,1 / 2,1 / 2)$. Fig. 4 shows the results of the CTSI and the proposed EUI $_{\mathrm{F}}$ by changing the concentration of total phosphorus $\left(x_{\mathrm{tp}}\right)$ from 17 to $2 \mu \mathrm{g} / \mathrm{L}$ and from 17 to $38 \mu \mathrm{g} / \mathrm{L}$, while the values of the other two variables are fixed. Sensitivity analysis shows that the result of the CTSI is linear, while the concentration of phosphorus changes from 17 to 

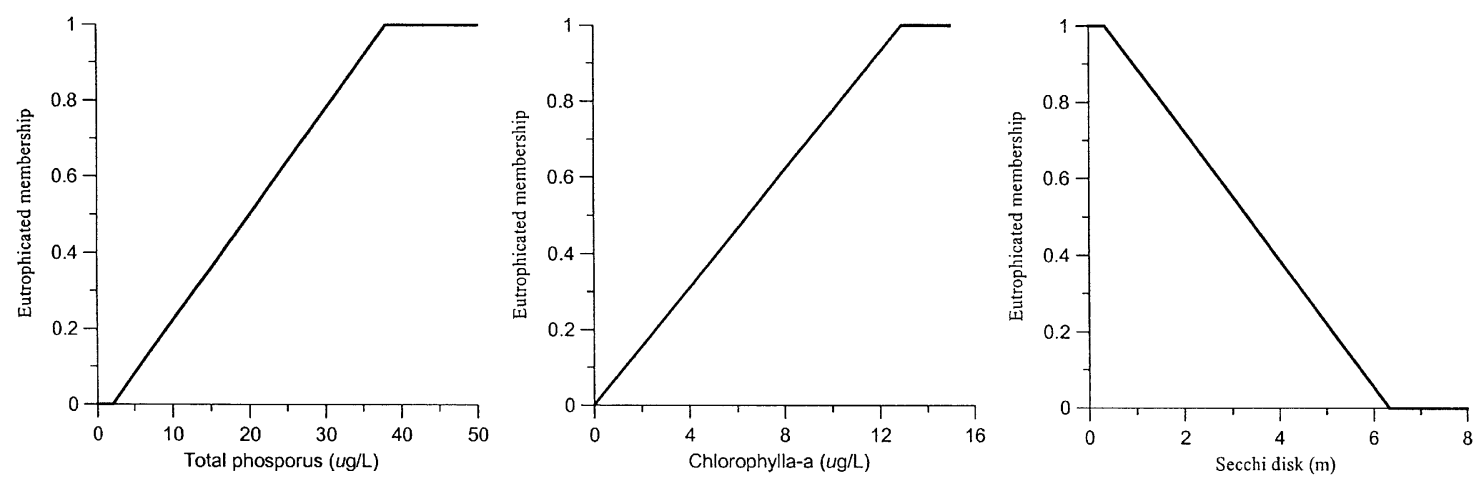

Fig. 3. The eutrophicated memberships of total phosphorous $(\mu \mathrm{g} / \mathrm{L})$, chlorophyll- $a(\mu \mathrm{g} / \mathrm{L})$, and Secchi disk (m).

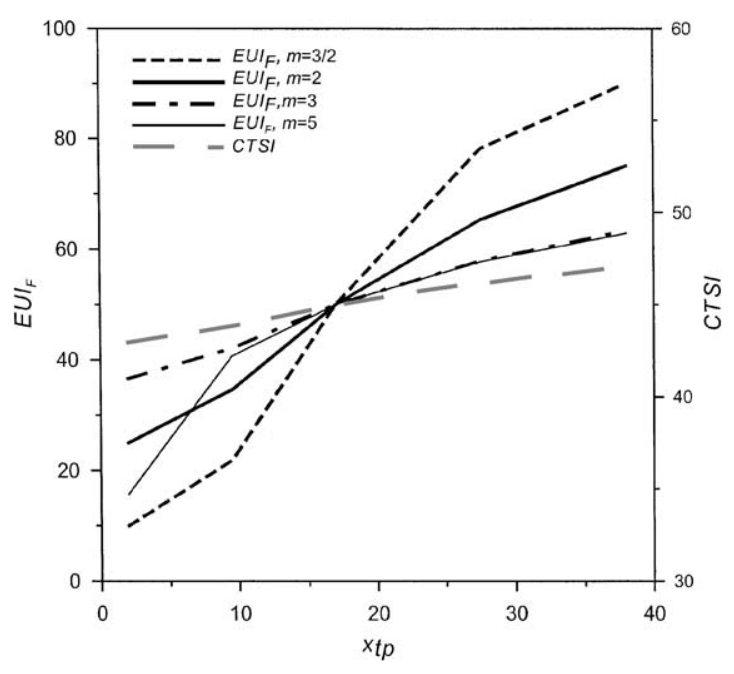

Fig. 4. The sensitivity analysis of EUI $\mathrm{F}_{\mathrm{F}}$ and the CTSI by initially setting the observation to $\vec{x}_{k}=\left(x_{\text {tp }}, x_{\text {chla }}, x_{\mathrm{sd}}\right)=$ $(17,4.34,2.83)$ and changing the concentration of total phosphorus.

$38 \mu \mathrm{g} / \mathrm{L}$ but varies sharply, while the concentration of total phosphorus changes from 17 to $2 \mu \mathrm{g} / \mathrm{L}$. Fig. 5 shows the results of EUI $\mathrm{F}_{\mathrm{F}}$ and the CTSI by changing the concentration of total phosphorus $\left(x_{\mathrm{tp}}\right)$, while the values of the other two variables are fixed to high eutrophic status, $x_{\text {chla }}=7.2 \mu \mathrm{g} / \mathrm{L}$ and $x_{\mathrm{sd}}=2 \mathrm{~m}$. The dotted black line represents the boundary between oligotrophication and mesotrophication referred to in Carson's Trophic State Index system. The CTSI estimates the object categorized as oligotrophic status when the concentration of total phosphorus drops to $2 \mu \mathrm{g} / \mathrm{L}$ but the other two variables are in high eutrophic states. The eclipsing occurs with the CTSI but does not occur over the results of $\mathrm{EUI}_{\mathrm{F}}$ with variety fuzziness index. Hence, in evaluating the trophic status, the proposed model, $\mathrm{EUI}_{\mathrm{F}}$, is more appropriate than the CTSI. Besides, from the sensitivity analysis, it is noted that the $\mathrm{EUI}_{\mathrm{F}}$

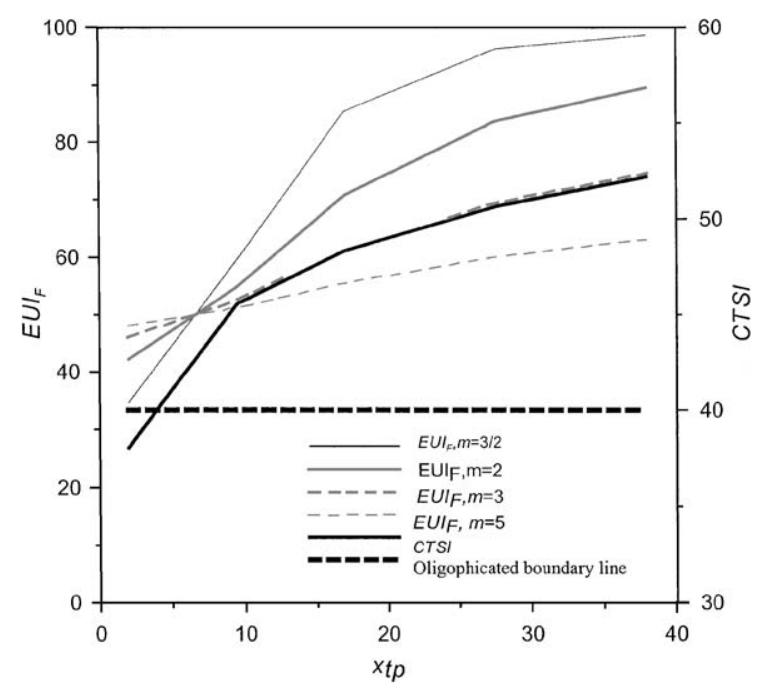

Fig. 5. The sensitivity analysis of $\mathrm{EUI}_{\mathrm{F}}$ and the CTSI by initially setting the observation to $\vec{x}_{k}=\left(x_{\mathrm{tp}}, x_{\mathrm{chla}}, x_{\mathrm{sd}}\right)=$ $(17,7.2,2)$ and changing the concentration of total phosphorus.

under the condition with fuzziness index $m=5$ and 3 is not appropriate because of low sensitivity.

The three specific eutrophic-ordered levels are defined for revealing eutrophicated performance against time into classification. A low value of the fuzziness index would be assigned to be associated with the three specific quality-ordered levels. The similarities between observed and assigned trophic status is derived as

$\mu_{k i}=\frac{1 /\left[\left\|\vec{f}_{k}-\vec{e}_{i}\right\|^{2 /(m-1)}\right]}{\sum_{j=1}^{3}\left[1 /\left(\left\|\vec{f}_{k}-\vec{e}_{j}\right\|^{2 /(m-1)}\right)\right]}$,

where $\quad \vec{e}_{1}=\left(f_{\mathrm{tp}}, f_{\text {chla }}, f_{\mathrm{sd}}\right)=(0,0,0), \quad \vec{e}_{2}=\left(f_{\mathrm{tp}}, f_{\text {chla }}\right.$, $\left.f_{\mathrm{sd}}\right)=(0.5,0.5,0.5)$, and $\vec{e}_{3}=\left(f_{\mathrm{tp}}, f_{\text {chla }}, f_{\mathrm{sd}}\right)=(1,1,1)$. The index is calculated by $\mathrm{EUI}_{\mathrm{F}}=\left(\mu_{k 1} \times 0+\mu_{k 2} \times\right.$ $\left.0.5+\mu_{k 3} \times 1\right) \times 100$. The term $\mu_{k 1}$ represents the similarity between the sampling data $\bar{x}_{k}$ and the oligotrophic 
status; $\mu_{k 2}$ indicates the similarity between the object and the mesotrophic status, and $\mu_{k 3}$ notes the similarity between the object and the eutrophic status. Referring to the diverse patterns of overall index models for quality evaluation shown in Fig. 2, the index curves pattern shown in Fig. 2 (B, IV) is desired. The two threshold values, $\mathrm{EUI}_{\mathrm{F}}=0$ and $\mathrm{EUI}_{\mathrm{F}}=50$, control the results of eutrophicated classification. The observation is defined as oligotrophication when the value of EUI $\mathrm{F}$ is zero. The observation is recognized as mesotrophication for the value of $\mathrm{EUI}_{\mathrm{F}}$ is greater than zero but less than or equal to 50 . As the value of $E_{\mathrm{F}}$ is over 50, the observation is categorized into eutrophication.

\subsection{A case study}

The Feitsui Reservoir was constructed in 1986 to supply water to metropolitan Taipei. A map of the eight regular sampling sites is shown in Fig. 6. The historical

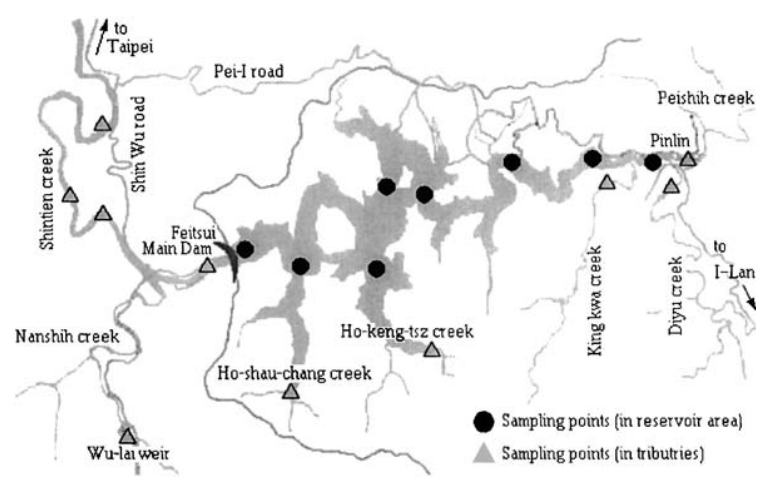

Fig. 6. Layout of water quality sampling points of the Feitsui Reservoir. regular monitored data from 1987 to 2003 were collected from the management agency of the Feitsui Reservoir. The monthly average over the eight sampling sites on the surface layer is used. Fig. 7 plots the eutrophicated performance of monthly time series and 12-month moving average of $\mathrm{EUI}_{\mathrm{F}}$ with the fuzziness index $m=$ 2. In addition, the monthly average and 12-month moving average of water inflows are shown in Fig. 6, where the unit CMM represents cubit meters per month. Two peaks are explored in temporal changes of the EUI $_{\mathrm{F}}$. One is in August $1988\left(\mathrm{EUI}_{\mathrm{F}}=91.02\right)$; the other is in December $1998\left(\mathrm{EUI}_{\mathrm{F}}=94.40\right)$. The unusual eutrophication of the Feitsui Reservoir during August 1988 is presumably due to the initial stage of dam operation in 1987. Chang and Wen (1998) indicated that internal nutrient loading of a newly built reservoir critically affects water quality during the initial period. The result of $\mathrm{EUI}_{\mathrm{F}}$ reveals that the nutrients were gradually exhausted and the eutrophic status of the Feitusi Reservoir gradually recovered from 1988 to 1995. Kuo (2002) stated that high percentage of annual total phosphorus leading to a reservoir is transported by high flows. The high-eutrophicated performance in December 1998 is reasonable because of high inflows that occurred in October 1998. Additionally, the temporal changes of the EUI $_{\mathrm{F}}$ typically follow an annual pattern. The eutrophic status is particularly significant in the end of summer and the beginning of autumn, just as Lu et al. (1999) indicated. Furthermore, the quality of the Feitsui Reservoir gradually decreased between 1996 and 2003, as the trend analysis shown in 12-month moving average shows. The construction of a highway between Yilan and Taipei, increased tourist activities in Pinglin, which is between the two cities, and the diverse rainfall intensity of recent years are the three

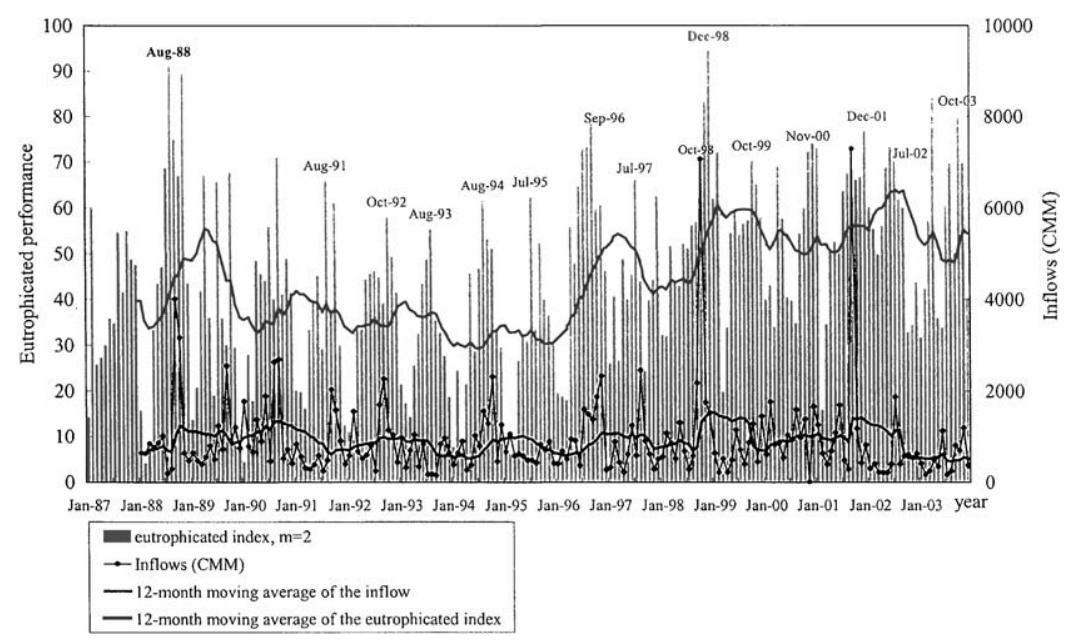

Fig. 7. The eutrophicated performance of $\mathrm{EUI}_{\mathrm{F}}$ with the fuzziness index $m=2$ and the water inflows from 1987 to 2003 on the Feitsui Reservoir. 


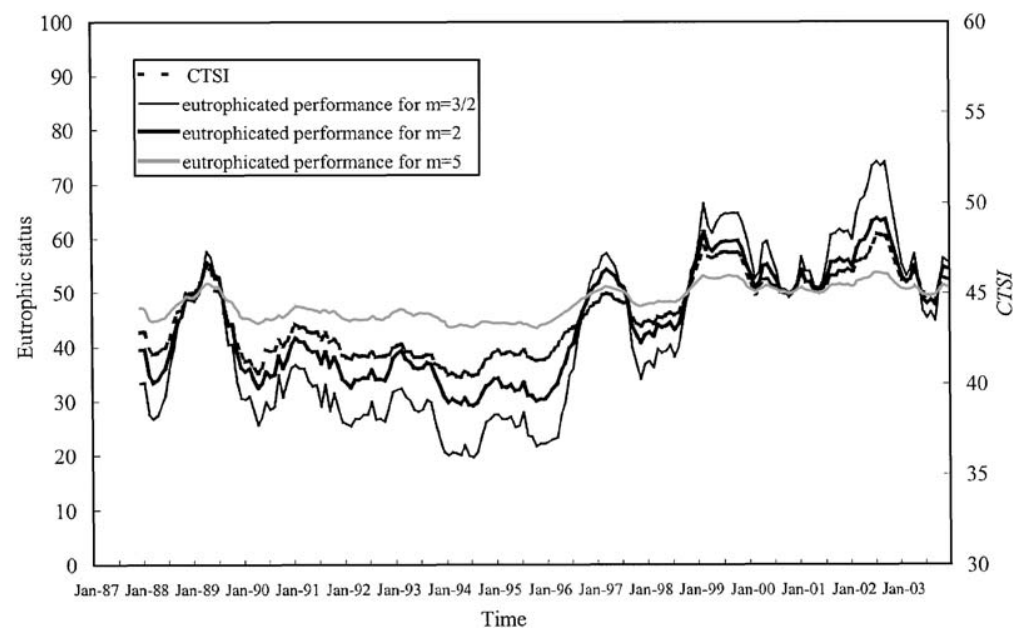

Fig. 8. The eutrophicated performance of 12-month moving average according to different fuzziness index for the Feitsui Reservoir.

Table 3

The data categorized to eutrophic status from 1987 to 2003

\begin{tabular}{|c|c|c|c|c|c|c|c|c|c|c|c|c|}
\hline & Jan & Feb & Mar & Apr & May & Jun & Jul & Aug & Sep & Oct & Nov & Dec \\
\hline 1987 & & Eutro- & & & & & & & & & & \\
\hline 1988 & & & & & & & & Eutro- & Eutro- & & Eutro- & \\
\hline 1989 & & & & & & & & & & Eutro- & & \\
\hline 1990 & & & & & & & & & & Eutro- & & \\
\hline 1991 & & & & & & & & Eutro- & & Eutro- & & \\
\hline 1992 & & & & & & & & & & Eutro- & & \\
\hline 1993 & & & & & & & & & & & & \\
\hline 1994 & & & & & & & & & & & & \\
\hline 1995 & & & & & & & & & & & & \\
\hline 1996 & & & & & & & & & Eutro- & & & \\
\hline 1997 & & & & & & & Eutro- & & & & & \\
\hline 1998 & & & & & & & & & & Eutro- & Eutro- & Eutro- \\
\hline 1999 & Eutro- & Eutro- & & & & & & & & Eutro- & Eutro- & \\
\hline 2000 & & & & Eutro- & & & & & & & Eutro- & Eutro- \\
\hline 2001 & Eutro- & & & & & & Eutro- & Eutro- & & Eutro- & Eutro- & Eutro- \\
\hline 2002 & Eutro- & Eutro- & & Eutro- & Eutro- & Eutro- & Eutro- & Eutro- & Eutro- & & & \\
\hline 2003 & & & & Eutro- & & & & & & Eutro- & Eutro- & \\
\hline
\end{tabular}

main reasons for the acceleration of the eutrophic status in the Feitsui Reservoir. Fig. 8 shows the curves of 12month moving averages for the CTSI and $\mathrm{EUI}_{\mathrm{F}}$. Although the proposed model under the condition with fuzziness index $m=2$ is recommended for consecutive trend analysis, the curve for $m=3 / 2$ is more significant than the curve for $m=2$ on displaying the changes of eutrophic status over a long period of time. Low sensitivity of the $\mathrm{EUI}_{\mathrm{F}}$ under the condition with fuzziness index $m=5$ is explored in Fig. 8 .

Table 3 records the results of categorizing the eutrophic status by employing the low-fuzziness index of $m=6 / 5$. The yearly eutrophic performance is irregular; and the duration of eutrophic status is longer than before 1998. Furthermore, two-thirds of 2002 was in eutrophic status. Heavy drought, strong rainfall, and warm weather in autumn would cause a high concentration of total phosphorus; consequently, heavy blooms developed in reservoirs. The management of reservoirs is increasingly important in preventing a worsening of water quality under the situation of climate change.

\section{Conclusion}

The idea of generating an index model for quality evaluation based on the formula of membership functions in the fuzzy c-means (FCM) clustering algorithm is 
introduced. This study explains how to manipulate the parameters of the similarity membership function for environment-quality analysis. On applying the index model to the eutrophicated analysis, sensitivity analysis discovers that eclipsing does not occur in the proposed model, unlike the CTSI. In addition, the case study shows the proposed index model is flexible and adaptable for diagnosing the eutrophic status of reservoir waters. Based on the results of this study, we conclude the following:

1. Stretching the values of the controlling parameters in the formula of similarity membership function results in diverse patterns of overall index models for quality evaluation. A consecutive overall index can be derived; a crisp classification analysis can also be performed.

2. A combined high-fuzziness index with excess specific quality-ordered levels leads the inconsistent results in consecutive analysis. The proposed index model switches from fuzzy theory to crisp theory as the value of observations coincides with the value of the specific quality levels.

3. The optimal $m$ value depends on the purpose of the index maker. The fuzziness index $m=2$ with two specific quality levels is generally recommended for consecutive analysis. For examining the quality or the eutrophic status over a long period of time, however, the value $m=3 / 2$ is more proper than $m=2$. In addition, a low-fuzziness index is used for classification evaluation; for example, the value $m=6 / 5$ is employed in this study.

4. The index detects that the eutrophic status of the Feitsui Reservoir has deteriorated rapidly in recent years because of climate changes and an increase in human activities around the watershed. The index can provide useful information to the reservoir manager.

\section{References}

Bezdek, J.C., 1980. A Convergence theorem for the fuzzy ISODATA clustering algorithms. IEEE Trans. Pattern Anal. Machine Intell. PAMI-2 (1), 1-8.

Carlson, R.E., 1977. A trophic state index for lakes. Limnol. Oceanog. 22 (2), 361-369.
Chang, S.P., Chuang, S.M., 2001. Eutrophication study of twenty reservoirs in Taiwan. Water Sci. Technol. 44 (6), 19-26.

Chang, S.P., Wen, C.G., 1998. Nutrient release from inundated land of tropical reservoir (Nanhua Reservoir, Taiwan). Water Sci. Technol. 37 (2), 325-332.

Chang, N.B., Chen, H.W., Ning, S.K., 2001. Identification of river water quality using the fuzzy synthetic evaluation approach. J. Environ. Manage. 63, 293-305.

Fadili, M.J., Ruan, S., Bloyet, D., Mazoyer, B., 2001. On the number of clusters and the fuzziness index for unsupervised FCA application to BOLD fMRI time series. Med. Image Anal. 5, 55-67.

Flores-Sintas, A., Cadenas, J.M., Martin, F., 1999. Membership functions in the fuzzy c-means algorithm. Fuzzy Sets System 101, 49-58.

Hammah, R.E., Curran, J.H., 1999. On distance measures for the fuzzy k-means algorithm for joint data. Rock Mech. Rock Eng. 32 (1), 1-27.

Kuo, J.T., 2002. Estimation of sediment and pollutant flux to a reservoir during a storm period. In: Proceeding of Advances in Hydro-Science and Engineering, vol. V, Warsaw University of Technology, Poland, 18-20 September.

Kung, H.T., Ying, L.G., Liu, Y.C., 1992. A complementary tool to water quality index: fuzzy clustering analysis. Water Resour. Bull. 28 (3), 525-533.

Liou, S.M., Lo, S.L., Hu, C.Y., 2003. Application of two-stage fuzzy set theory to river quality evaluation in Taiwan. Water Res. 37, 1406-1416.

Lu, R.S., Lo, S.L., 2002. Diagnosing reservoir water quality using self-organizing maps and fuzzy theory. Water Res. 36 (9), 2265-2274.

Lu, R.S., Lo, S.L., Hu, J.Y., 1999. Analysis of reservoir water quality using fuzzy synthetic evaluation. Stoch. Environ. Res. Risk Assess. 13, 327-336.

Melcher, D., Matthies, M., 1996. Application of fuzzy clustering to data dealing with phytotoxicity. Ecol. Model. $85,41-49$.

Organization for Economic Co-operation and Development, 1982. Eutrophication of waters. OECD Publications Office, Paris.

Silvert, W., 1997. Ecological impact classification with fuzzy sets. Ecol. Model. 96, 1-10.

Silvert, W., 2000. Fuzzy indices of environmental conditions. Ecol. Model. 130, 111-119.

Tao, Y., Xinmiao, Y., 1998. Fuzzy comprehensive assessment, fuzzy clustering analysis and its application for urban traffic environment quality evaluation. Transp. Res. 3 (1), 51-57.

Whitman, I., Fahringer, D., 1973. An environmental evaluation system for water resource planning. Water Resour. Res. 9 (3), 523-535. 